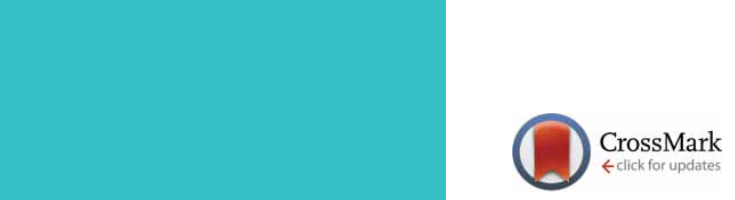

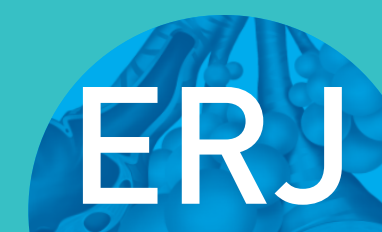

open research
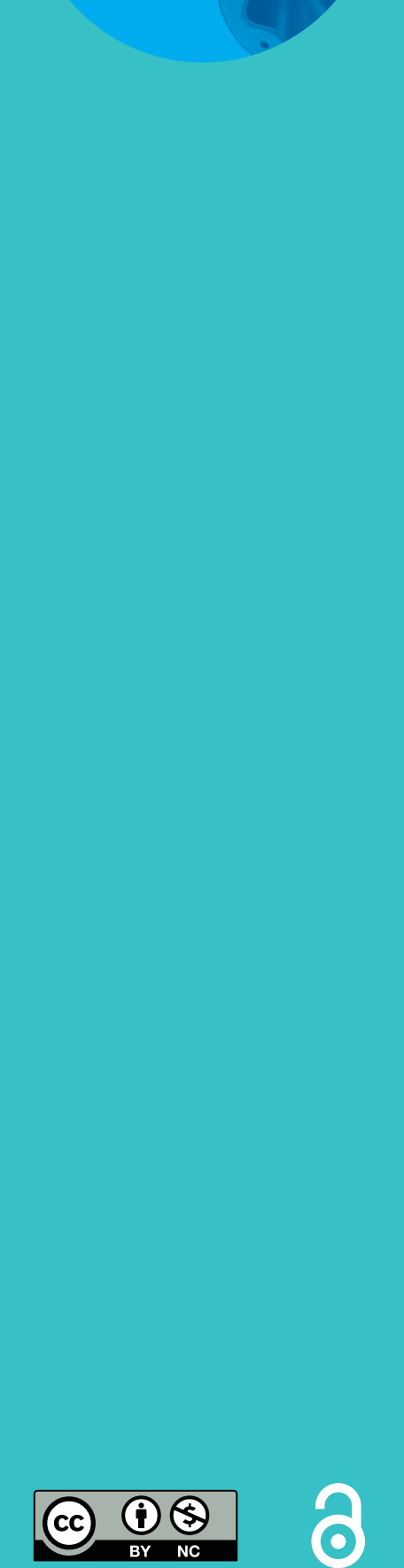

\section{ERS International Congress 2020: highlights from the Respiratory Infections assembly}

\author{
Stefan Frent ${ }^{1}$, Cristina Calarasu $\mathbb{1}^{2}$, Kseniia Suska ${ }^{3}$, Kateryna Gashynova ${ }^{3}$ and \\ Holly Keir ${ }^{4}$
}

Affiliations: ${ }^{1}$ Dept of Pulmonology, University of Medicine and Pharmacy Timisoara, Timisoara, Romania. ${ }^{2}$ Dept of Medical Specialities, University of Medicine and Pharmacy Craiova, Craiova, Romania. ${ }^{3}$ State Institution "Dnipropetrovsk Medical Academy of the Ministry of Health of Ukraine", Dnipro, Ukraine. ${ }^{4}$ Scottish Centre for Respiratory Research, Ninewells Hospital and Medical School, University of Dundee, Dundee, UK.

Correspondence: Cristina Calarasu, Department of Medical Specialities, University of Medicine and Pharmacy Craiova, Petru Rares Street no. 2, Craiova, Dolj 200349, Romania. E-mail: calarasu.cristinadyahoo.com

ABSTRACT In the coronavirus disease 2019 (COVID-19) pandemic year 2020, the 30th European Respiratory Society (ERS) International Congress took place for the first time in a fully virtual format. Despite the challenging nature of the task to create and deliver an online event of this size and scope, it turned out to be a great success, welcoming over 33000 delegates to the specially designed online platform and offering more than 450 scientific and educational sessions. Somewhat predictably, this year's ERS International Congress dedicated a full day to the topic of COVID-19, highlighting that infection with severe acute respiratory syndrome coronavirus 2 (SARS-CoV-2) is a respiratory disease that is particularly important this year. In this article, the Early Career Members of the Assembly 10 (Respiratory Infections and Tuberculosis) review some of the most interesting sessions including presentations and posters on respiratory infections and tuberculosis that were deemed as important.

@ERSpublications

This is a comprehensive summary of several interesting sessions from \#ERSCongress 2020 including presentations and posters on respiratory infections and tuberculosis that were deemed as important https://bit.ly/2OtrYXH

Cite this article as: Frent S, Calarasu C, Suska K, et al. ERS International Congress 2020: highlights from the Respiratory Infections assembly. ERJ Open Res 2021; 7: 00091-2021 [https://doi.org/ 10.1183/23120541.00091-2021].

Received: 4 Feb 2021 | Accepted after revision: 25 Feb 2021

Copyright $\odot$ The authors 2021. This version is distributed under the terms of the Creative Commons Attribution NonCommercial Licence 4.0. For commercial reproduction rights and permissions contact permissions@ersnet.org 


\section{COVID-19 recapitulation}

T. Welte opened the ERS Virtual Congress 2020 with a keynote lecture entitled "COVID-19: recapitulation". At the time of this presentation, there were 27 million SARS-CoV-2 infections and 875000 deaths recorded worldwide. This is a novel virus, and T. Welte stressed the challenges of day-to-day changes in knowledge in terms of diagnosis, treatment and preventative measures. In early September, patient numbers were beginning to decrease in many countries and the case fatality rate was reducing from $10-15 \%$ to $3-5 \%$ worldwide [1].

T. Welte gave an overview of the COVID-19 pandemic, with the first case of COVID-19 being reported in Wuhan, China, on the December 8, 2019. Infection spread through the city of Wuhan, causing the healthcare system to be overwhelmed and subsequently a large increase in mortality. This was followed by cases being detected in the larger Hubei region; however, as the healthcare system remained intact mortality was 10-times lower than during the initial Wuhan outbreak. A strict lockdown policy was imposed, which rapidly brought down the infection rate, with preventative messages still on a high level and virus transmission low as of September $2020[2,3]$.

The virus arrived in Europe in January 2020, believed to have been exacerbated by Italian Fashion Week due to increased travel by international visitors. Several "superspreader" events, such as a football game in Milan on February 21, contributed to the spread of the virus in Europe. A breakdown in healthcare systems in a number of countries, including Italy, Spain, France and the UK led to an increase in daily mortality caused, in part, by COVID-19 but also from a lack of normal healthcare services for other conditions [4]. The delayed arrival of the pandemic in Germany resulted in an improved response to the outbreak. Unlike a number of other European countries, Germany saw no increase in daily mortality during the first wave of the pandemic. T. Welte emphasised that the instigation of an earlier lockdown, a more robust healthcare system, differences in living conditions and an improved knowledge of appropriate treatments were key factors in improving the response to the pandemic.

T. Welte's conclusions underlined the importance of scientific and clinical advice in combating the pandemic, while emphasising that strategies and decisions must be adaptable in this rapidly evolving situation.

\section{New developments in pandemic medicine: an update from the PREARE research project}

The aim of this session was to describe the latest developments in antiviral therapy for influenza and the growing problem of arbovirus infection in Europe, to evaluate the rationale for adaptive trials investigating critical care interventions in a pandemic, and to describe a collaborative strategy for increasing clinical and research capacity in Europe for pandemic care.

C. Butler reviewed the latest results for the Antivirals for influenza-Like Illness? An rCt of Clinical and Cost-effectiveness in primary CarE (ALIC ${ }^{4} \mathrm{E}$ ) of oseltamivir, an antiviral therapy for influenza. A Cochrane collaboration review [5] which summarised the previous ROCHE funded trials of oseltamivir found that treatment alleviated symptoms in adults at $16.8 \mathrm{~h}$ compared with placebo. However, the review also contained criticism of trial design, including a lack of diagnostic definitions for complications of influenzae and low numbers of paediatric patients and patients with co-morbidities. A BMJ editorial [6] concluded that a large definitive clinical trial that focussed on understudied groups by an independent body was required to assess whether adding oseltamivir treatment in primary care was truly worthwhile.

$\mathrm{ALIC}^{4} \mathrm{E}$ [7] was designed to meet these criteria. The trial encompassed 21 networks over 15 countries in 207 primary care practices. 3259 patients were randomised, 14\% of whom were children aged under 12 years. $15 \%$ of subjects had a chronic comorbidity, and an increase in distribution of illness severity and a range of time that subjects could be symptomatic for compared to previous trials. Trial results found that time to recovery was shorter in participants receiving oseltamivir (hazard ratio $1.29,95 \%$ Bayesian credible interval (BCrI) 1.20-1.39) overall. The estimated absolute mean benefit from oseltamivir was 1.02 days (95\% BCrI 0.74-1.31) overall, and ranged from 0.70 (95\% BCrI 0.30-1.20) in patients younger than 12 years, with less severe symptoms, no comorbidities, and shorter previous illness duration to 3.20 ( $95 \%$ BCrI 1.00-5.50) in patients aged $\geqslant 65$ years who had more severe illness, comorbidities and longer previous illness duration. To conclude, although the average benefit for many patients is modest, older patients who are more severely unwell may have an improvement in recovery time of 2-3 days.

L. Sigfrid discussed the work of ISARIC (International Severe Acute Respiratory and emerging Infection Consortium) [8]. ISARIC is a global federation of clinical research member networks involved in patient-based research, with a mission to prevent illness and deaths from infectious disease outbreaks. The consortium aims to enable timely, robust, standardised patient-based research as the heart of an evidence-based response to epidemics and pandemics. Made up of 50 networks in 132 countries, these 
pre-existing networks are a key enabler for rapid standardised response. ISARIC has previously responded to other pandemics in collaboration with the World Health Organization (WHO). Together, they have developed a Clinical Characterisation Protocol (CCP), designed to respond to any emerging pathogen that is of public health interest. CCP is designed to have a flexible tiered approach, which is adaptable depending on site resources in order to standardise patient data. Tier 0 is for data collection only, tier 1 for data collection and admission samples and tiers 2-3 are for data collection and serial sampling depending on resources. Associated with the protocol is a range of collection tools which standardise data collection. CCP protocols have been activated in response to other emerging pathogens, including Middle East respiratory syndrome coronavirus (2012), influenza H7N9 (2013), ebolavirus (2014), monkeypox (2018), tick-borne encephalitis virus (2019) and was initiated in January 2020 in response to COVID-19.

In January 2020, data from China on the COVID-19 outbreak was reviewed and an updated core COVID-19 case report form launched, later followed by a RAPID COVID-19 case report form which included additional modules for sites able to collect more complex data in critical care. Protocols are open access: sites are able to sign up to capture electronic data in a standardised format. By August 25, more than 96000 patient data records were on the database, across 562 sites and 42 countries.

L. Sigfrid gave an overview of the emerging data; 81705 (82\%) individual had laboratory-confirmed SARS-CoV-2 (as of August 6, 2020). 57\% were male with a median age of 71 years. The most common symptoms recorded were cough (62\%), fever (61\%), shortness of breath (59\%) and fatigue/malaise (36\%). Other common symptoms included altered consciousness, nausea/vomiting and diarrhoea. The most common comorbidities were hypertension (46\%), chronic cardiac disease $(30 \%)$, chronic kidney disease $(16 \%)$ and diabetes $(16 \%)$, and $14.5 \%$ of subjects had no recorded co-morbidities. Median onset of symptoms after admission was 4 days, with the median length of hospital stay being 9 days. Of patients with an outcome recorded, $58 \%$ recovered, $28 \%$ of patients died (62\% male, $38 \%$ female) and this rose to $34 \%$ in patients admitted to ICU. In terms of treatment, $81 \%$ of patients received antibiotics, $65 \%$ received oxygen therapy and $17 \%$ received steroids. A subset analysis was done in the UK cohort assessing the impact of COVID-19 paediatric admissions. The analysis included 651 children, with a median age of 4.6 years, with $42 \%$ having one or more comorbidities. The death rate was very low at $1 \%$, with all patients having profound comorbidities [9]. To conclude, L. Sigfrid presented the launch of the ISARIC follow up studies, with an invitation for other sites to join the consortium.

G. Carson, in her review entitled "What's next - preparing for the ongoing COVID response and future pandemics", explored what the pandemic has taught us so far. The COVID-19 pandemic put healthcare workers in a unique position, of responding, recovering and preparing for resurgences simultaneously. G. Carson discussed the work of the Global Preparedness Monitoring Board (GPMB), which was created in 2018 after the ebola outbreak shed light on the major gaps in political, research public health systems" capacity to prevent, detect and respond to health crises from local to global levels. A report by Herten-Crabi et al. [10] examined 26 recommendations for dealing with global health emergencies, focussing on global coordination and governance. A key finding of the report was the "Global Safety Net" which highlighted the importance of strengthening global preparedness and response.

COVID-19 has had immense social, health and economic costs and the pandemic has demonstrated the need for a collective global response. Social determinants of health play a role in the increased risk faced by certain groups. Effective response to outbreaks should consider multiple disciplines, including health care, research, social impact and political policy. To conclude, G. Carson highlighted WHO "Sustainable Development Goals (SDGs)" as a pathway to improving global cohesion and response, as well as the importance of working with communities, avoiding politisation, implementing a systems-based approach across sectors, securing funding for public health and scenario planning to tackle COVID-19 globally.

\section{Post-COVID session}

The long-term effects of COVID-19 are relatively unknown; researchers are exploring parallels with other disease and trialling long-term management options. The aim of this session was to discuss the current care pathways, rehabilitation options, long-term impact on the lung and palliative care for patients.

I. Vogiatzis started the session by highlighting the effects of COVID-19 on physical, functional and emotional health. It has been well documented that patients with COVID-19 report physical functional disability during hospital admission and after discharge. Pulmonary function is also reduced at admission but typically improves after 6 weeks, although some degree of restrictive alterations still persists [11]. In addition to physical symptoms, there is a substantial prevalence of anxiety and depression among patients with COVID-19 [12]. ERS has issued guidance on the hospital and post-hospital phases of COVID-19 [13], which has been compiled by 93 experts from 23 countries. The proposed care pathway covers guidance from bedside rehabilitation in the hospital setting through to discharge and follow-up. The guidance 
recommends formal assessment at 6-8 weeks of: physical and emotional function, patient core outcomes, respiratory function, exercise capacity and need for rehabilitation. Based on the outcomes of this assessment, patients should be offered support to aid their recovery, including comprehensive rehabilitation, muscle strengthening programmes, nutritional guidance and psychological support.

The British Thoracic Society (BTS) has also issued guidance on rehabilitation to support recovery of the post-COVID population [14]. The BTS care pathway recommends that, within 6-8 weeks, patients should be referred to pulmonary rehabilitation team for assessment and subsequent follow up. I. Vogiatzis highlighted that with physical distancing rules in many places due to the pandemic, there is a need for some rehabilitation and assessment to happen remotely. Virtual consultations can be utilised to assess a range of core outcomes to facilitate the implementation of recovery programs. Virtual sessions should aim to give patients a programme to follow at home, including psychological support, nutritional guidance, advice on physical activity and diaries to track symptoms and recovery. In response to COVID-19, healthcare professional consultations should be employed to assess key outcomes and provide recovery programmes for patients.

S. Singh summarised the impact of COVID-19 on patients, which treatable traits are involved and how rehabilitation pathways may aid recovery. A study of 143 patients in an Italian cohort found that patients who had recovered from COVID-19 continued to report persistence of at least one symptom, in particular fatigue, breathlessness, joint pain and chest pain [15]. Additionally, patients have reported a profound reduction in exercise capacity post-COVID, even in younger patient populations. In terms of psychological health, post-traumatic stress disorder, anxiety and depression have all been reported by patients. Current rehabilitation programmes should be updated to reflect the new post-COVID population and the specific challenges they present. Programmes should consider including psychological and fatigue support, speech and language therapy for patients who have been ventilated and nutritional support as well as pulmonary rehabilitation.

F. Blasi discussed the long-term impact of COVID-19 on the lungs. Post-discharge, patients have reported symptoms, such as breathlessness, fatigue, anxiety and depression, and as many as one in two patients have symptoms at follow-up [15]. There is growing evidence that SARS-CoV-2 infection can lead to pulmonary fibrosis [16]. In a study of 110 post-COVID patients, more than one-third of patients discharged developed fibrotic abnormalities, $47 \%$ had impaired diffusing capacity of the lungs for carbon monoxide and $25 \%$ had reduced total lung capacity [17]. It has been speculated that acute and chronic inflammation in COVID-19 results in alveolar epithelium damage, which stimulates over expression of pro-inflammatory cytokines. This leads to fibroblasts and myofibroblast activation, followed by excessive deposition of collagen in the extracellular matrix resulting in pulmonary fibrosis. There is currently little evidence for which therapies are most appropriate to treat these patients, although potential treatments include steroids, current idiopathic pulmonary fibrosis (IPF) treatments and anti-viral medications. Finally, F. Blasi highlighted that COVID-19 infection is associated with ongoing myocardial inflammation, independent of pre-existing conditions, severity and overall course of acute illness.

D. Janssen led the final session discussing palliative care for COVID-19 patients. There are specific challenges to COVID-19 palliative care: patient deterioration can be fast and unpredictable, healthcare systems are under immense burden due to the pandemic, patients require isolation, family visits are restricted or carried out remotely and communication is hampered by personal protective equipment (PPE). In a study of 101 patients receiving palliative care for COVID-19, the reported symptom burden was predominately breathlessness (66\%), agitation (43\%), drowsiness (35\%), delirium (24\%) and pain (23\%) [18]. In order to guide healthcare professionals (HCP) and improve palliative care for patients an ERS taskforce was formed. 90 international experts completed an online survey on 14 potential recommendations, with at least $70 \%$ agreement on directionality required for consensus recommendations. Recommendations have been provided for 14 domains including: advance care planning, palliative treatment of breathlessness, need for training in optimising patient-clinician communication while wearing PPE, training for remote clinician-family communication, involvement in HCPs trained in palliative care, involvement of HCPs providing spiritual and psycho-social care, bereavement support for patients and support for HCPs. This multi-national consensus can be accessed through the European Respiratory Journal [19].

\section{The future of bronchiectasis management}

The aims of this session were to describe the effects of early life, including demographic, inflammatory and genetic factors, on the development of bronchiectasis; to review recent randomised studies and observational data that are leading to improvements in the management of bronchiectasis; to describe current options for the management of Pseudomonas aeruginosa pulmonary infections in patients with 
bronchiectasis; and to describe possible future treatments for bronchiectasis in adults. The session was chaired by G.G.U. Rohde (Frankfurt, Germany) and E. Polverino (Barcelona, Spain).

K. Ramsey (Bern, Switzerland) opened the session by highlighting the role of altered airway mucus viscosity in the pathogenesis of bronchiectasis [20] and the relatively low number of studies characterising mucus composition in bronchiectasis patients [21]. Recently published results of an experiment conducted by RAMSEY et al. [22] at the University of North Carolina at Chapel Hill, USA, on the airway mucus concentration in patients with non-cystic fibrosis bronchiectasis were further presented. The aim of the study was to assess concentration, composition and biophysical properties of bronchiectasis mucus in sputum samples collected from subjects enrolled in the BLESS trial [23] compared with mucus obtained from healthy control subjects.

K. Ramsey and colleagues found a hyperconcentration of mucus in bronchiectasis patients compared to healthy subjects, with MUC5B and MUC5AC being the major secreted mucins. Mucus hyperconcentration and altered biophysical properties (increased osmotic pressure and increased complex viscosity) are further responsible for impaired mucociliary and cough clearance. The researchers also found a positive correlation between mucus concentration and bronchiectasis extent score, but no correlation with forced expiratory volume in $1 \mathrm{~s}$, and the neutrophilic inflammation being driven by high bacterial loads of all pathogens, not just Pseudomonas spp. Previous studies have demonstrated neutrophil elastase to be a key driver of the disease [24-26] by inducing persistent muco-obstruction and triggering interleukin (IL)- $1 \beta$-driven inflammatory cascade, leading ultimately to the development of bronchiectasis [27]. Hypertonic saline reduced mucin concentration by $25 \%$ in induced sputum samples [22]; however, clinical trials assessing effectiveness of mannitol and hypertonic saline in patients with bronchiectasis showed mixed results. Both mucoactive drugs demonstrated an improvement in the quality of life, but no significant effect on the number of exacerbations [28].

Ramsey concluded the presentation by proposing a reliable and relatively easy to use biomarkers for identification of patients who would benefit from muco-modulatory treatment: sputum dry to wet weight ratio.

A. O'Donnell from Georgetown University (WA, USA) gave a perspective on the current state-of-the-art management of bronchiectasis. While the cure of bronchiectasis by structural damage reversal or mortality reduction are still aspirational goals, the reduction of exacerbations, symptoms control, improvement in quality of life, preservation of lung function and treatment of the underlying disease may be feasible with the current therapeutic means. Bronchiectasis remains a highly heterogeneous disease, and currently available evidence supports the concept of multimodality treatment tackling each pathogenic pathway of the "vicious vortex" [20]

An organised multimodality approach in A. O'Donnell's view consists in the identification of a potentially treatable underlying disease, the assessment of disease severity and an individualised treatment tailored to patient's needs, including airway clearance therapies, anti-inflammatory treatments, antibiotics and " $\mathrm{N}$ of 1 " interventions.

Treatable causes of bronchiectasis include genetic disorders (e.g. cystic fibrosis, $\alpha_{1}$-antitrypsin deficiency (AATD), primary ciliary dyskinesia (PCD)), immune defects (e.g. immunoglobulin deficiency, HIV infection) and structural abnormalities (airway obstruction, aspiration)). A. O'Donnell stressed the importance of identification and correct management of these underlying conditions.

Airway clearance therapy addresses to the airway structural dysfunction with the aim to overcome the failure of mucus clearance. Active interventions include physical exercise (pulmonary rehabilitation), mucus drainage techniques, the use of mechanical devices and pharmacological therapies [29, 30]. Hypertonic saline 7\% improved lung function and quality of life in non-cystic fibrosis bronchiectasis patients [31], bronchodilators and $N$-acetyl cysteine have an unknown efficacy, while mannitol and rhDNase proved to be ineffective [32, 33].

Anti-inflammatory therapies include inhaled corticosteroids (ICS) and long-term use of macrolides. While ICS should be used with caution after a judicious assessment of the risk/benefit ratio due to increased risk of non-tuberculous mycobacterial infection [34], there is mounting evidence for the role of long-term treatment with macrolides in exacerbation reduction [35]. Specific interventions like surgery [36], lung transplantation [37] or anti-reflux procedure may be used in selected patients, on a case by case basis.

The management of chronic airway infection in bronchiectasis patients was reviewed by J. Chalmers from the University of Dundee (UK) in the light of several recently published meta-analyses. Chronic airway infection, particularly with $P$. aeruginosa, is associated with increased risk of hospitalisation and increased mortality [38]. Current ERS guidelines for the management of adult bronchiectasis with $\geqslant 3$ exacerbations per year recommend long-term inhaled antibiotic for patients with P. aeruginosa infection and long-term macrolide for non-P. aeruginosa infection [28]. 
A recently published individual participant data meta-analysis of three randomised controlled trials of macrolide antibiotics in adult patients with bronchiectasis conducted by CHALMERS et al. [35] demonstrated that long-term macrolide antibiotics significantly reduced the frequency of exacerbations and prolonged the time to first exacerbation, and was associated with improved quality of life as measured by the Saint George's Respiratory Questionnaire. Subgroup analyses revealed the highest level of benefit for patients with a history of $P$. aeruginosa infection and one or two exacerbations per year.

The efficacy of inhaled antibiotics in the reduction of pulmonary exacerbations is supported by inconsistent data from randomised clinical trials [39]. A systematic review and meta-analysis of 16 randomised controlled trials of inhaled antibiotic use in adult patients with bronchiectasis and chronic respiratory tract infections showed a small but significant reduction in exacerbation frequency, without significant improvement in quality of life [40]. In order to better identify the responders to inhaled antibiotic therapy, SiBILA et al. [41] conducted two prospective studies of adults with bronchiectasis and a post hoc analysis of a randomised trial of inhaled aztreonam. They found a significant improvement in respiratory symptoms score (QOL-B-RSS) in favour of aztreonam compared with placebo in the subgroup of patients with high bacterial load. This finding was further confirmed by a re-analysis of Orbit 3 and 4 studies conducted by CHALmers et al. [42], showing significant improvements in respiratory symptoms during the on-treatment periods which were lost during off-treatment periods. These newly accumulating data may prompt an update of current guidelines for the management of bronchiectasis patients with frequent exacerbations, as suggested by LASKA and CHALMERS [43] in a recent editorial.

J. Chalmers concluded the talk by briefly mentioning the promising data from the WILLOW study, which demonstrated that reduction of neutrophil serine protease activity with brensocatib in patients with bronchiectasis is associated with reduction in exacerbation risk and prolonged time to first exacerbation [44]. Thus, the future of bronchiectasis management may not be antibiotics.

F. Blasi (Milan, Italy) in the final talk of the session focussed on the concept of treatable traits in bronchiectasis, emphasising that it could be a useful strategy to help clinicians consider the many different aspects that must be addressed for the appropriate clinical management of patients with bronchiectasis [45]. F. Blasi further discussed two of the challenges frequently encountered by the physicians caring for patients with bronchiectasis: identification of underlying diseases and understanding the inflammatory pattern.

Despite using the current international guidelines suggested bundle of tests to investigate bronchiectasis aetiology [46], the majority of patients are labelled as having "idiopathic bronchiectasis". In order to increase the chances to identify a treatable underlying etiology, FRANCESCHI et al. [47] proposed an extensive bundle of tests, additionally including immunoglobulin (Ig)G subclasses, lymphocytes subpopulations, HIV test, PICADAR score and nasal nitric oxide measurement for the screening of PCD, sweat test and second level cystic fibrosis transmembrane conductance regulator genetic analysis, AATD genetics and screening tests for connective tissue diseases. Using the extended bundle, the authors were able to significantly increase the percentage of patients with a treatable aetiology and to identify the presence of "subclinical" genetic alterations which could presumably find clinical expression by a cumulative effect, after reaching a specific threshold.

Sputum neutrophil elastase is a marker of disease activity in bronchiectasis patients and associates with increased risk of exacerbations, lung function decline and mortality [26]. The ability of a novel semi-quantitative lateral flow device to provide a point of care assessment of neutrophil elastase activity was tested by SHommark et al. [48], who demonstrated a good correlation between high neutrophil elastase activity as measured by the device and a significant increase in exacerbation frequency.

Another study conducted by SHoEmark et al. [49] identified three inflammatory endotypes in patients with clinically stable bronchiectasis: 1) neutrophilic inflammation; 2) eosinophilic and epithelial inflammation; and 3) systemic inflammation, which may represent different "treatable traits". Unpublished data from an Italian cohort of 700 bronchiectasis patients bring additional evidence for the existence of a T2-high endotype characterised by high levels of blood eosinophils, total IgE and oral nitric oxide. Finally, a group of researchers from Hanover, Germany were able to demonstrate significant improvements in lung function, quality of life and sputum production and a trend towards reduced exacerbation rate after 6 months of treatment with anti-IL5 $(n=12)$ and anti-IL-5R $\alpha(n=9)$ in 21 patients with clinically significant bronchiectasis and features of refractory disease despite optimised therapy [50].

\section{Airway infection: the microbiome and beyond}

This poster session included ten studies providing novel insights on the airways' microbiome and its influence on the course of respiratory diseases. 
Meszaros et al. [51] studied the incidence and economic burden of under-recognised respiratory infections in older adults, such as pertussis. It was reported that the incidence of diagnosed pertussis in England between 2009 and 2018 was higher among patients aged 50 years or more with comorbid asthma than in patients 50 years or older without a history of asthma. It was also noted that given the under-diagnosis of pertussis in adults further research is warranted on the potential health and economic impact of pertussis in asthmatics.

In turn, VAN DEN STEEN et al. [52] assessed the seroprevalence of Bordetella pertussis in COPD patients aged 40-85 years from the AERIS (Acute Exacerbations and Respiratory InfectionS in COPD) study in the UK between 2009 and 2018. This study showed a seroprevalence of Pertussis spp. in more than 13\% of COPD patients which turned out to be higher than in the UK Clinical Practice Research Datalink and indicated a substantial under-diagnosis of pertussis, warranting improved estimates and evaluation of its impact on COPD outcomes.

MANn et al. [53] presented safety and disease outcomes from a novel respiratory syncytial virus (RSV) challenge study in 60 to 75 -year olds. RSV Memphis37b was administered intranasally to patients, and then longitudinal disease profiles of older subjects were compared to a historical cohort of "younger" healthy subjects. This novel RSV challenge of 60-75-year-olds was considered safe and induced an appropriate level of disease, allowing for the assessment of vaccines and drugs targeted at 60-75-year-old population.

VAN BRAECKEL et al. [54] presented preliminary clinical data from the international multicentre collaboration aiming to improve chronic pulmonary aspergillosis (CPA) knowledge and patient care: CPAnet registry. Data reflecting real-world clinical practice was collected from several international centres and included CPA phenotype, comorbidities, treatment, outcomes and follow-up. Other CPA centres were invited to join this initiative.

JABEEN et al. [55] compared performance of Illumina MiSeq, Nanopore sequencing, and RT-qPCR (quantitative reverse transcription polymerase chain reaction) on total DNA extracts against culture/ MALDI-TOF for analysis of induced sputum samples from well-phenotyped severe asthma patients. Haemophilus influenzae was identified as the dominant bacterial species by metagenomic sequencing using MiSeq, Nanopore and was validated with $H$. influenzae plasmid-based RT-qPCR assay, allowing for characterisation of this clinical phenotype.

KEIR et al. [56] presented the results of a microbiome analysis of the sputum from patients with stable COPD and during exacerbations, performed by 16S rRNA (ribosomal ribonucleic acid) sequencing. There were no statistically significant differences between stable and exacerbation microbiome profiles as a whole. However, a subgroup analysis of bacterial exacerbations (with positive sputum culture) revealed an association with significant loss of diversity and divergent changes in the overall composition of the microbiome.

The next presentation was devoted to the prevalence and genetic adaptation of persistent P. aeruginosa infections in patients with COPD. EKLÖF et al. [57] conducted whole-genome sequencing study of $P$. aeruginosa strains sampled longitudinally from sputum cultures in 23 patients enrolled in a randomised controlled trial in Denmark. The results indicated a relationship between $P$. aeruginosa infections and genetically defined mucociliary clearance defects (GDMCD), as 37 P. aeruginosa genes were independently mutated in two or more lineages, suggesting a positive selection for adaptive mutations which are also important for the persistence of infection in patients with GDMCD.

The results of the largest multicentre evaluation of the COPD mycobiome were presented by TiEw et al. [58] who examined COPD mycobiomes in 380 patients from Singapore, Malaysia and Scotland, based on mortality and occurrence of serum specific-IgE against fungi. In patients with frequent exacerbations, systemic specific-IgE responses against Aspergillus spp., Penicillium spp. and Curvularia spp., as well as lower survival rates were noted, which, according to the authors, allows for COPD risk stratification based on airway mycobiomes.

In connection with the widespread use of macrolides in the therapy of patients with frequent exacerbations of chronic respiratory disease, NARAYANA et al. [59] studied airway microbiomes and corresponding resistomes using deep sequencing metagenomic approaches from airway specimens of 85 individuals with and without severe asthma, COPD and bronchiectasis. Macrolide resistance was found to be dominant in a "core" airway resistome, but also a high prevalence of $\beta$-lactam, fluoroquinolone and tetracycline resistance genes was reported. The greatest resistance was observed in COPD and bronchiectasis patients and was found to be independent of disease status or prior antibiotic exposure. In addition, Streptococcus spp. and Actinomyces spp. were revealed as potential microbial reservoirs of macrolide resistance including the ermX, ermF and $m s r D$ genes. 
Mu et al. [60] presented the results of a prospective evaluation of novel nanopore-based metagenomic sequencing, which was used for rapid detection of bacterial pathogens in patients with lower respiratory tract infections in comparison with those isolated by microbiology tests, then the discrepancies were checked by real-time PCR. The sensitivity and specificity of the nanopore sequencing were $96.6 \%$ and $88.0 \%$, respectively. Moreover, nanopore metagenomics identified pathogens in 63 out of 161 culture-negative samples, and $50(79.4 \%)$ of them were verified. These results are promising for the application of this new technique in the clinical practice, in order to achieve a more accurate diagnosis of respiratory infections.

\section{E-poster sessions: Tuberculosis and society, non-tuberculous mycobacteria and latent tuberculosis infection, the young, the old and extrapulmonary tuberculosis, multidrug-resistant tuberculosis}

From the following session, we picked some of the most interesting posters presented on tuberculosis to highlight the need for continued research in order to find better diagnostic and therapeutic tools for tuberculosis in a pandemic year.

In one study, Bussi et al. [61] used human induced-pluripotent stem cell derived macrophages (iPSDM) together with high-content live-cell imaging, extracellular flux analysis and unbiased metabolomics to investigate mitochondrial dynamics, Mycobacterium tuberculosis intracellular replication and mitochondrial metabolism. Surprisingly, M. tuberculosis did not induce significant changes in the mitochondrial area, length or width during the first $48 \mathrm{~h}$ of infection, however, the metabolic profile of infected macrophages showed an increase in oxygen consumption and extracellular acidification rate after $48 \mathrm{~h}$ of infection. Conclusions stressed that macrophage metabolic reprogramming is required for the control of $M$. tuberculosis replication and that, unlike other intracellular pathogens, changes in host cell metabolism induced by $M$. tuberculosis might correlate with disruption of mitochondrial function rather than morphology.

VLADIMIRSKY et al. [62] had an interesting presentation about a new M. tuberculosis culture format using the lytic D29 mycobacteriophages for accelerated determination of drug sensitivity of $M$. tuberculosis to first- and second-line anti-tuberculosis drugs in clinical isolates obtained after initial cultivation. When comparing this new method with the Mycobacteria Growth Indicator Tube Bactec system in 108 clinical isolates, the coincidence of results in determining drug sensitivity was demonstrated in up to $98 \%$ of cases. In conclusion, using lytic mycobacteriophage to determine drug sensitivity for M. tuberculosis may be rapid (up to 5 days), simple to implement and cost effective.

PANOva et al. [63] studied an application of next-generation sequencing (NGS) to detect M. tuberculosis resistance to first- and second-line anti-tuberculosis drugs aiming to find key drug resistance-associated mutations of $M$. tuberculosis and compare it with phenotype-based methods. Drug resistance was evaluated through single nucleotide polymorphisms identification in resistance-associated genes $(r p s L$, $r R N A, k a t G$, inhA, rpoB, embB, pncA, rpsA, gyrA, gyrB, rrs, eis). NGS has been shown to be a highly sensitive method for detecting mutations associated with resistance to isoniazid, rifampicin, ofloxacin, levofloxacin, moxifloxacin (100\%) and streptomycin (96.7\%). Sensitivity values for detection of resistance to ethambutol and kanamycin were lower (87.5\% and 88.9\%). Low sensitivity was observed for pyrazinamide (29.4\%), amikacin and capreomycin (60.0\%). Specificity was more than $90 \%$ for all anti-tuberculosis drugs.

SHARMa et al. [64] presented a six-case study on phenotyping and treatment of endobronchial tuberculosis (EBTB) diagnosed on histopathology samples obtained via bronchoscopic (FOB) biopsy. EBTB was found to mainly affect young females ( $<30$ years), often involving left main bronchus. To prevent recurrent lobar or complete lung collapse in spite of adequate tuberculosis treatment, the authors used balloon dilatation, mucosal incisions and stents, but two patients required pneumonectomy in the end. Conclusion of the author was that identification of this phenotype of tuberculosis and early application of bronchoscopy in diagnosis and management of EBTB can prevent significant debilitation or lung tissue loss.

AL-SALIHI et al. [65] studied non-communicable (NCDs) comorbidities of tuberculosis in Iraq using data from the national anti-tuberculosis drug resistance survey (DRS) finalised in 2015. Comorbid NCDs for tuberculosis include diabetes, smoking, malnutrition and chronic lung disease. The data review of 1160 study participants revealed the following prevalence rates of NCDs: $33.8 \%$ were smokers, $17.6 \%$ had a history of anaemia in the last 6 months, $24.4 \%$ had diabetes, $19.2 \%$ were underweight, $3.6 \%$ had asthma, $5.6 \%$ used steroid treatment on presentation and only $1.6 \%$ had severe chronic diseases (chronic pulmonary disease/severe renal disease). The study evidenced that the first four conditions were related to tuberculosis within 6 months prior to diagnosis. 
RusAKOVA et al. [66] tried to identify predictors of death in patients with multi-drug resistant tuberculosis (MDR-TB) and HIV in a retrospective cohort study with 1-year follow-up of $130 \mathrm{MDR}-\mathrm{TB} / \mathrm{HIV}$ patients, of whom, 31 (23.9\%) patients had died. Several potential predictors of death were analysed: age, sex, body mass index (BMI), history of previous treatment, adherence to treatment, $\mathrm{CD}^{+}$level in $1 \mu \mathrm{L}$, drug resistance, liver size, haemoglobin and protein levels in $\mathrm{g} / \mathrm{L}^{-1}$, hepatitis history, alcohol and drug addiction, homelessness. Median values were calculated for the subgroups of patients who survived after one year of follow-up (L) or who died within 1 year of follow-up (D). Statistically significant differences were obtained for: homelessness $(\mathrm{OR}=4.8 ; \mathrm{p}=0.05)$, alcohol and drug addiction (for alcoholism, $\mathrm{OR}=2.4 ; \mathrm{p}=0.05$; for drug addiction, $\mathrm{OR}=2.8 ; \mathrm{p}=0.02)$, history of being lost to follow-up ( $\mathrm{OR}=4.7 ; \mathrm{p}=0.05)$, BMI $(\mathrm{L}=29 ; \mathrm{D}=19$; $\mathrm{p}=0.001)$, number of $\mathrm{CD}^{+}(\mathrm{L}=334 ; \mathrm{D}=117 ; \mathrm{p}=0.001)$, haemoglobin level $(\mathrm{L}=133 ; \mathrm{D}=122 ; \mathrm{p}=0.03)$ and the total protein $(\mathrm{L}=77 ; \mathrm{D}=22 ; \mathrm{p}=0.006)$. Subsequent logistic regression analysis revealed that the most important predictors of death for MDR-TB/HIV patients include history of being lost to follow-up, low $\mathrm{CD}^{+}$, and low BMI.

A very interesting method to treat tuberculosis patients was presented by Hidalgo et al. who studied pulmonary surfactant as a new way to deliver anti-tuberculosis drugs. The interfacial properties and lipid composition of pulmonary surfactant are ideal solutions to solubilise and transport hydrophobic drugs by surfing the respiratory surface, targeting alveoli and phagocytic cells. The authors proposed to use pulmonary surfactant to solubilise and transport the anti-tuberculosis drug, bedaquiline, over a respiratory air-liquid interface and demonstrated that pulmonary surfactant can incorporate bedaquiline preserving the interfacial performance as analysed in a Langmuir trough and a captive bubble surfactometer. Also, they measured the appearance of the drug at the recipient trough by mass spectrometry (using a special in vitro setup), and confirmed that pulmonary surfactant transports bedaquiline interfacially. The authors observed a prominent antibiotic effect of pulmonary surfactant/bedaquiline formulations suggesting the synergistic therapeutic effects of pulmonary surfactant/bedaquiline combinations compared with the drug alone, and highlighted the potential of pulmonary surfactant as an anti-tuberculosis carrier [67].

\section{Concluding remarks}

In this comprehensive summary of several interesting sessions, the authors tried to share a glimpse of the impressive amount of scientific information delivered at the ERS International Congress annually. We encourage our readers to deepen their knowledge according to the field of interest by further reading of the cited sources and we also hope that we have stimulated the desire for participation at future ERS scientific events.

Author contributions: S. Frent reported on the session "The future of bronchiectasis management"; C. Calarasu reported on the e-poster sessions "Tuberculosis and society", "Non-tuberculous mycobacteria and latent tuberculosis infection", "The young, the old and extrapulmonary tuberculosis" and "Multidrug-resistant tuberculosis"; K. Suska and K. Gashynova reported on the session "Airway infection: the microbiome and beyond"; and H. Keir reported on the sessions "COVID-19 recapitulation", "New developments in pandemic medicine: an update from the PREARE EU research project" and "Post-COVID session". All the authors reviewed and agreed on the content of the article.

Conflict of interest: None declared.

\section{References}

Medicine JHUa. Coronavirus Resource Center. 2020.

2 Wu Z, McGoogan JM. Characteristics of and important lessons from the Coronavirus Disease 2019 (COVID-19) outbreak in China: summary of a report of 72314 cases from the Chinese Center for Disease Control and Prevention. JAMA 2020; 323: 1239-1242.

3 Li Q, Guan X, Wu P, et al. Early transmission dynamics in Wuhan, China, of novel coronavirus-infected pneumonia. N Engl J Med 2020; 382: 1199-1207.

4 Magnani C, Azzolina D, Gallo E, et al. How large was the mortality increase directly and indirectly caused by the COVID-19 epidemic? an analysis on all-causes mortality data in Italy. Int J Environ Res Public Health 2020; 17: 3452

5 Jefferson T, Jones MA, Doshi P, et al. Neuraminidase inhibitors for preventing and treating influenza in healthy adults and children. Cochrane Database Syst Rev 2014; 2014(4): CD008965.

6 Weber JT, Nicoll A, Bridges CB, et al. The truth about Tamiflu? Neuraminidase inhibitors in pandemic A/H1N1 flu. BMJ 2010; 340: c130.

7 Butler CC, van der Velden AW, Bongard E, et al. Oseltamivir plus usual care versus usual care for influenza-like illness in primary care: an open-label, pragmatic, randomised controlled trial. Lancet 2020; 395: 42-52.

8 Dunning JW, Merson L, Rohde GGU, et al. Open source clinical science for emerging infections. Lancet Infect Dis 2014; 14: 8-9.

9 Swann OV, Holden KA, Turtle L, et al. Clinical characteristics of children and young people admitted to hospital with covid-19 in United Kingdom: prospective multicentre observational cohort study. BMJ 2020; 370: m3249.

10 Herten-Crabb A, McDonald B, Sigfrid L, et al. The state of governance and coordination for health emergency preparedness and response. 2019.

11 Fumagalli A, Misuraca C, Bianchi A, et al. Pulmonary function in patients surviving to COVID-19 pneumonia. Infection 2021; 49: 153-157. 
12 Zhang J, $\mathrm{Lu} \mathrm{H}$, Zeng $\mathrm{H}$, et al. The differential psychological distress of populations affected by the COVID-19 pandemic. Brain Behav Immun 2020; 87: 49-50.

13 Spruit MA, Holland AE, Singh SJ, et al. COVID-19: interim guidance on rehabilitation in the hospital and post-hospital phase from a European Respiratory Society and American Thoracic Society-coordinated International Task Force. Eur Respir J 2020; 56: 2002197.

14 Singh SJ, Barradell AC, Greening NJ, et al. British Thoracic Society survey of rehabilitation to support recovery of the post-COVID-19 population. BMJ Open 2020; 10: e040213.

15 Carfi A, Bernabei R, Landi F. Persistent symptoms in patients after acute COVID-19. JAMA 2020; 324: $03-05$.

16 Spagnolo P, Balestro E, Aliberti S, et al. Pulmonary fibrosis secondary to COVID-19: a call to arms? Lancet Respir Med 2020; 8: 750-752.

17 Mo X, Jian W, Su Z, et al. Abnormal pulmonary function in COVID-19 patients at time of hospital discharge. Eur Respir J 2020; 55: 2001217.

18 Lovell N, Maddocks M, Etkind SN, et al. Characteristics, symptom management, and outcomes of 101 Patients With COVID-19 referred for hospital palliative care. J Pain Symptom Manage 2020; 60: e77-e81.

19 Janssen DJA, Ekström M, Currow DC, et al. COVID-19: guidance on palliative care from a European Respiratory Society international task force. Eur Respir J 2020; 56: 2002583.

20 Flume PA, Chalmers JD, Olivier KN. Advances in bronchiectasis: endotyping, genetics, microbiome and disease heterogeneity. Lancet 2018; 392: 880-890.

21 Sibila O, Suarez-Cortin G, Rodrigo-Troyano A, et al. Secreted mucins and airway bacterial colonisation in non-CF bronchiectasis. Respirology 2015; 20: 1082-1088.

22 Ramsey KA, Chen ACH, Radicioni G, et al. Airway mucus hyperconcentration in non-cystic fibrosis bronchiectasis. Am J Respir Crit Care Med. 2020; 201: 661-670.

23 Serisier DJ, Martin ML, McGuckin MA, et al. Effect of long-term, low-dose erythromycin on pulmonary exacerbations among patients with non-cystic fibrosis bronchiectasis: the BLESS randomised controlled trial. JAMA 2013; 309: 1260-1267.

24 Keir HR, Fong CJ, Dicker AJ, et al. Profile of the ProAxsis active neutrophil elastase immunoassay for precision medicine in chronic respiratory disease. Expert Rev Mol Diagn 2017; 17: 875-884.

25 Oriano M, Gramegna A, Terranova L, et al. Sputum Neutrophil Elastase associates with microbiota and P. aeruginosa in bronchiectasis. Eur Respir J 2020; 57: 2000769.

26 Chalmers JD, Moffitt KL, Suarez-Cuartin G, et al. Neutrophil elastase activity is associated with exacerbations and lung function decline in bronchiectasis. Am J Respir Crit Care Med 2017; 195: 1384-1393.

27 Boucher RC. Muco-obstructive lung diseases. N Engl J Med 2019; 380: 1941-1953.

28 Polverino E, Goeminne PC, McDonnell MJ, et al. European Respiratory Society guidelines for the management of adult bronchiectasis. Eur Respir J 2017; 50: 1700629.

29 Nicolson CH, Holland AE, Lee AL. The bronchiectasis toolbox - a comprehensive website for the management of people with bronchiectasis. Med Sci (Basel) 2017; 5: 13

30 O'Neill K, O'Donnell AE, Bradley JM. Airway clearance, mucoactive therapies and pulmonary rehabilitation in bronchiectasis. Respirology 2019; 24: 227-237.

31 Kellett F, Robert NM. Nebulised 7\% hypertonic saline improves lung function and quality of life in bronchiectasis. Respir Med 2011; 105: 1831-1835.

32 Bilton D, Tino G, Barker AF, et al. Inhaled mannitol for non-cystic fibrosis bronchiectasis: a randomised controlled trial. Thorax 2014; 69: 1073-1079.

33 O'Donnell AE, Barker AF, Ilowite JS, et al. Treatment of idiopathic bronchiectasis with aerosolised recombinant human DNase I. rhDNase Study Group. Chest 1998; 113: 1329-1334.

34 Brode SK, Campitelli MA, Kwong JC, et al. The risk of mycobacterial infections associated with inhaled corticosteroid use. Eur Respir J 2017; 50: 1700037.

35 Chalmers JD, Boersma W, Lonergan M, et al. Long-term macrolide antibiotics for the treatment of bronchiectasis in adults: an individual participant data meta-analysis. Lancet Respir Med 2019; 7: 845-854.

36 Mitchell JD. Surgical treatment of pulmonary nontuberculous mycobacterial infections. Thorac Surg Clin 2019; 29: 77-83.

37 Rusanov V, Fridman V, Wille $\mathrm{K}$, et al. Lung transplantation for cystic fibrosis and non-cystic fibrosis bronchiectasis: a single-center experience. Transplant Proc 2019; 51: 2029-2034.

38 Chalmers JD, Goeminne P, Aliberti S, et al. The bronchiectasis severity index. An international derivation and validation study. Am J Respir Crit Care Med 2014; 189: 576-585.

39 Haworth CS, Bilton D, Chalmers JD, et al. Inhaled liposomal ciprofloxacin in patients with non-cystic fibrosis bronchiectasis and chronic lung infection with Pseudomonas aeruginosa (ORBIT-3 and ORBIT-4): two phase 3 , randomised controlled trials. Lancet Respir Med 2019; 7: 213-226.

40 Laska IF, Crichton ML, Shoemark A, et al. The efficacy and safety of inhaled antibiotics for the treatment of bronchiectasis in adults: a systematic review and meta-analysis. Lancet Respir Med 2019; 7: 855-869.

41 Sibila O, Laserna E, Shoemark A, et al. Airway bacterial load and inhaled antibiotic response in bronchiectasis. Am J Respir Crit Care Med 2019; 200: 33-41.

42 Chalmers JD, Cipolla D, Thompson B, et al. Changes in respiratory symptoms during 48 -week treatment with ARD-3150 (inhaled liposomal ciprofloxacin) in bronchiectasis: results from the ORBIT-3 and -4 studies. Eur Respir J 2020; 56: 2000110.

43 Laska IF, Chalmers JD. Treatment to prevent exacerbations in bronchiectasis: macrolides as first line?. Eur Respir J 2019; 54: 1901213 .

44 Chalmers JD, Haworth CS, Metersky ML, et al. Phase 2 trial of the DPP-1 inhibitor brensocatib in bronchiectasis. N Engl J Med 2020; 383: 2127-2137.

45 Boaventura R, Sibila O, Agusti A, et al. Treatable traits in bronchiectasis. Eur Respir J 2018; 52: 1801269.

46 Hill AT, Sullivan AL, Chalmers JD, et al. British Thoracic Society Guideline for bronchiectasis in adults. Thorax 2019; 74: Suppl 1, 1-69.

47 Franceschi E, Aliberti S, Seia M, et al. An extensive bundle of tests is needed to detect treatable causes of bronchiectasis (Bx). Eur Respir J 2018; 52: OA3270. 
Shoemark A, Cant E, Carreto L, et al. A point of care neutrophil elastase activity
severity, airway infection and risk of exacerbation. Eur Respir J 2019; 54: OA4947. Shoemark A, Smith A, Giam A, et al. Inflammatory molecular endotypes in bronchiectasis. Eur Respir J 2019; 54 PA2170.

50 Rademacher J, Konwert S, Jan Fuge J, et al. Anti-IL5 and anti-IL5R $\alpha$ therapy for clinically significant bronchiectasis with eosinophilic endotype: a case series. Eur Respir J 2020; 55: 19013.

51 Aris E, Akpo EI, Bhavsar A, et al. The burden of pertussis in adults with asthma: a retrospective database study in England. Eur Respir J 2020; 56: Suppl 64, 4926.

52 Mukherjee P, Cheuvart B, Baudson N, et al. Seroprevalence of Bordetella pertussis in chronic obstructive pulmonary disease (COPD) patients. Eur Respir J 2020; 56: Suppl 64, 4927.

53 Mann A, Kalinova M, Catchpole A, et al. Experimental respiratory syncytial virus infection in adults 60-75 years. Eur Respir J 2020; 56: Suppl 64, 4928.

54 Braeckel EV, Acker LV, Salzer HJF, et al. CPAnet registry - an international chronic pulmonary aspergillosis registry. Eur Respir J 2020; 56: Suppl 64, 4929.

55 Jabeen M, Street T, Foster D, et al. Applying modern molecular microbiological techniques to identify treatable chronic bacterial airway infection in severe asthma. Eur Respir J 2020; 56: Suppl 64, 4930.

56 Keir HR, Dicker A, Lonergan M, et al. Clinical endotypes of exacerbation are associated with differences in microbial composition and diversity in COPD. Eur Respir J 2020; 56: 2000391.

57 Eklöf J, Misiakou MA, Sivapalan P, et al. Persistence and genetic adaptation of Pseudomonas aeruginosa in patients with COPD. Eur Respir J 2020; 56: Suppl 64, 4932

58 Tiew PY, Dicker A, Keir HR, et al. A high-risk airway mycobiome characterises frequent COPD exacerbators. Eur Respir J 2020, 56: Suppl 64, 4933.

59 Narayana JK, Aogáin MM, Xu KLJ, et al. Co-occurrence analysis relates a macrolide resistome to the pulmonary microbiome in chronic respiratory disease. Eur Respir J 2020; 56: Suppl 64, 4934.

$60 \mathrm{Mu} \mathrm{S}, \mathrm{Hu} \mathrm{L}$, Zhang Y, et al. Prospective evaluation of nanopore-based metagenomic sequencing for rapid detection of bacterial pathogens in patients with lower respiratory tract infections. Eur Respir J 2020; 56: Suppl 64, 4935.

61 Bussi C, Dos Santos MS, Bernard EM, et al. Mycobacterium tuberculosis modulates mitochondrial function in human macrophages. Eur Respir J 2020; 56: Suppl. 64, 2807.

62 Vladimirsky M, Lapenkova M, Alyapkina Y. The use of the lytic D29 mycobacteriophages for accelerated determination of the drug sensitivity of tuberculosis mycobacteria to first and second-line anti-TB drugs in clinical isolates obtained after initial cultivation. Eur Respir J 2020; 56: Suppl. 64, 5296.

63 Panova A, Vinokurov A, Lagutkin D, et al. Application of next-generation sequencing to detect MTB resistance to first- and second-line anti-TB drugs. Eur Respir J 2020; 56: Suppl 64, 1605.

64 Sharma S, Ghoshal AG, Krishnan S, et al. Phenotyping and treatment of endobronchial tuberculosis: a case series. Eur Respir J 2020; 56: Suppl. 64, 2800

65 Al-Salihi L, Mankhi A. Non-communicable comorbidities of Tuberculosis. Eur Respir J 2020; 56: Suppl. 64, 1440.

66 Rusakova L, Saenko A, Sterlikov S. Predictors of death in patients with MDR-TB and HIV. Eur Respir J 2020; 56 Suppl. 64, 1599 .

67 Hidalgo A, Perez-Gil J, Lehr CM. Pulmonary surfactant: a Trojan Horse to deliver anti-TB drugs. Eur Respir J 2020; 56: Suppl. 64, 465. 